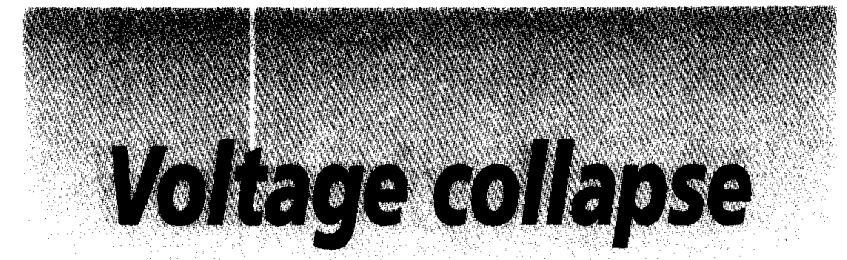

\title{
An engineering challenge
}

Mariesa L. Crow and Bernard C. Lesieutre

Recent blackouts in major cities throughout the world, including Tokyo and New York City, have raised concerns about a relatively new phenomenon in power system behavior: voltage collapse. This type of event is characterized by a loss of control of the voltage levels in a power system. Often, the initial voltage decay is so gradual, system operators are un-aware that the declining voltages are symptomatic of

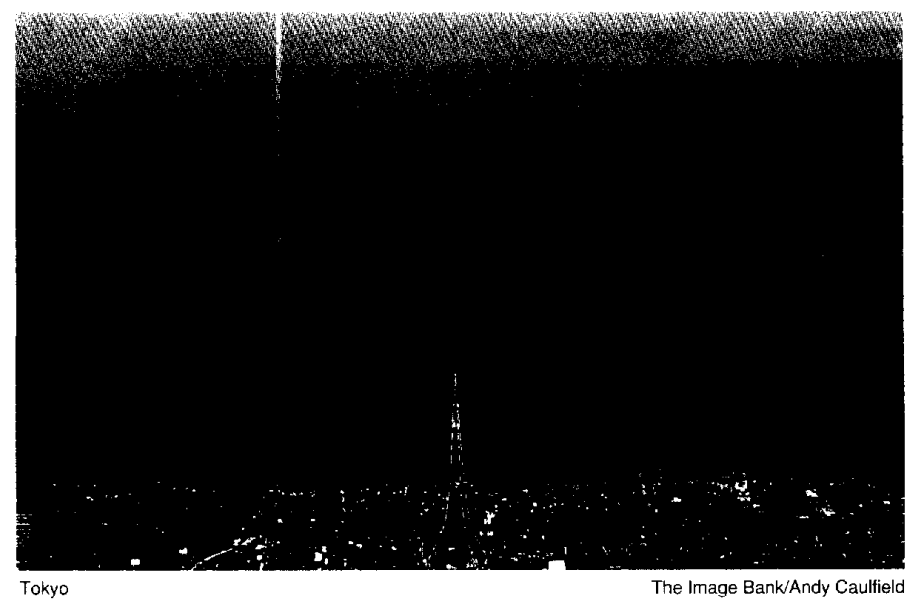

The Image Bank/Andy Caulfield

small fraction of the additional power required by 2010. The largest shortfall of power will occur in highly populated areas, where older facilities are being retired but not replaced. To compensate for this deficiency, the necessary additional power must be imported from other utilities as well as non-utility sources, often over considerable distances. This causcs considerable stress on the remaining facilities, as well as the power a collapse, until it is imminent. Then it is too late. The system operators are unable to avoid the catastrophic conciusion.

Although all of the precise mechanisms that affect voltage collapse have not yet been identified, voltage instabilities are known to occur when the power system is operating under stressed conditions. Recent changes in the political, economical, social, and environmental aspects of power systems have increased the frequency of operating under increasingly stressed conditions. This raises serious concerns about the ability of the U.S. power grid to safely and economically meet the load demands forecasted into the next century.

The Energy Information Agency (EIA) of the United States Department of Energy predicts that the demand for electrical energy in the United States will increase at an average rate of between 1.3 and $1.9 \%$ per year until the year 2010 . Although this rate is low compared to historic growth ratcs, in today's operating atmosphere, this growth rate still poses serious challenges.

Due to this low growth rate, there is a reluctance among power producing utilities to expand their generation capabilities. In addition, the recently enacted Clean Air Aot has caused further hesitation to build new fossil fuel plants. Many of the older coal burning plants are being forced out of operation since it is not economically feasible to retrofit most of them with pollutant reducing devices called "scrubbers."

Similarly, because of economic and political reasons, no new nuclear power reactors have been ordered s nce 1978. In fact, in the past two years, three reactors have been "retired." Of the twelve reactors that will have their operating licenses reviewed before 2010 , it is expected that only five of the twelve will be renewed.

Although it is expected that nuclear power will again be economically feasible in the early $2000 \mathrm{~s}$, it will on ly make up a

system transmission network. In many cases, the presently available resources are being taxed to their operating limits.

In addition to generation difficulties, the import and export of power through the transmission network may cause a multitude of additional problems. The transmission network is interconnected and extremely complex. Presently this network is being used in ways for which it was not originally designed. The power that utilities routinely import and export, often flows through other utilities' transmission networks. This makes it difficult to monitor and control.

Presently, more than $40 \%$ of the power generated by major utilities is sold to other utilities. Moreover, power transfer among utilities has more than doubled in the last twenty years. This "wheeling" of power is expected to increase as utilities-unable to meet their own consumer power demands-will choose to import power from utilities with excess generation.

In order to cope with the increased burden, the transmission capabilities must be expanded. However, citing health and environmental reasons, there has often been resistance to approve the building of new transmission lines. For example, the state of Florida enacted a moratorium against the building of new transmission lines until a formal public policy limiting electric and magnetic field levels near transmission lines was passed. This new regulation is estimated to cost Florida utilities an additional $\$ 100$ million to $\$ 5$ billion over the next 30 years. Similar measures have been proposed in other states.

These combined circumstances have resulted in many occurrences of voltages collapse in the United States and worldwide. A "classical" example occurred on July 23, 1987 in Tokyo. Japan. The forecasted temperature was $91^{\circ} \mathrm{F}$ and the forecasted power consumption level was 38,500 MW (MegaWatts). The day turned out to be hotter than expected with tem- 
peratures reaching a steamy $102^{\circ} \mathrm{F}$. By 11:00 a.m., the forecasted load had been adjusted to $40,000 \mathrm{MW}$.

At approximately 1:00 p.m., the load power demand increased at a rate of 400 $\mathrm{MW} / \mathrm{min}$ (setting a record for load power demand increase) and the line voltage began to sag. By 1:20 p.m. the line voltage was more than $20 \%$ lower than the normal operating voltage. At this point, protective relays shut down parts of the city with loss of power to 2.8 million customers.

The adjusted forecast for the load power exceeded Tokyo Electric Power Company's (TEPCO) maximum generation capabilities. To compensate for this high level of demand, TEPCO tried to import power from neighboring utilities. Despite this action, the system was not able to deliver the required power to the load center of Tokyo. The mismatch in demand and supply caused the voltage levels to decrease until a widespread blackout occurred.

This is a prime example of a highly stressed system. It is exemplified by a high level of load power coupled with a large amount of long distance power transmission. The situation in Tokyo was further exacerbated by the record increase in power demand.

\section{The mechanics}

In many of the recorded incidences of voltage collapse, the system voltage decreased slowly as the system demand increased, until a critical point was reached. At this point, any slight increase in demand yielded a large decrease in voltage, until the demand could no longer be satisfied. When the generating capabilities could not match the load demand, the system dynamics caused the voltage to drop quickly and irreversibly. In the classical scenario in which the load demand is largely unaffected to voltage variations, the critical point is often referred to as the point of maximum power transfer (PMPT).

Although voltage collapse is complex, some behavior can be understood by studying the system steady state voltage profile under various loading levels. The behavior of the voltage levels is illustrated by a typical power-voltage (PV) curve (Fig. 1), in which the voltage at a bus (node) is plotted as a function of the system real power demand. This curve is multi-valued.

To understand this better, recall that zero load power is achieved by either an open circuit (zero current) or a short circuit (zero voltage) ("OC" and "SC" in
Fig. 1). The right most tip of this curve is the PMPT. A voltage collapse is said to have occurred when the system reaches the PMPT and the voltage decreases very suddenly in the vicinity of the PMPT. When no more power can be transmitted through the network to the load, the system dynamics react to cause the voltage to decrease rapidly.

The lower portion of the curve is included for completeness, but due to the lower voltages associated with each demand level, operation on this part of the curve seldom occurs. Typically, the critical voltage level occurs well below the minimum range of acceptable voltages. Thus, corrective action usually occurs long before the system voltage reaches the PMPT. PV curves of this type are used frequently by utilities to study the effect of varied system loading patterns on voltage, and to predict the occurrence of potentially low voltages.

One commonly used means of controlling the voltage behavior is to add reactive power support by switching in shunt capacitors at low voltage buses. This action serves to increase the voltage level by decreasing the apparent load power (the magnitude of the complex power) at that bus. This also raises the PMPT to a greater real power demand level.

The voltage response with reactive power support is shown in Fig. 2. While reactive power support to control the voltage is productive over a wide range of system demand, in some cases this action may lead to a false sense of security. That is, the voltage collapse can occur with less prior notification. This can be seen clearly from Fig. 2 . With reactive power support at the load bus, the voltage remains within the acceptable range for the majority of the system loading levels, even after the system (without any reactive power support) has reached the critical loading point. However, shortly after the voltage falls below the acceptable range, it reaches the new critical loading point. If the demand is increasing rapidly, there may not be time enough to take corrective measures. The voltage rapidly collapses.

Figures 1 and 2 show the voltage levels calculated by varying the degree of loading. In reality, however, the voltage response of a system depends not only on the degree of loading (in MVA), but also on the load characteristics. A typical distribution load will contain a mixture of residential and industrial loads.

Industrial loads are generally com-
Public relations

Cold weather, as well as hot con cause problems. On January 19, 1994 demand for power on the United States' east coast kept rising in response to the freezing temperatures As a result, rolling blackouts -20 minutes to an hour in length-were initiated so nuclear plants and other power stations wouldn't shut down on their own causing a total blackout However, another problem resulted according to The New York Times.... one of communications.

The night before (Jan. 18), PSE\&G's communications department had started preparing faxes and prerecorded messages asking customers to cut back on power usage According to The New York Times, at 7:15 a.m. the next moming, PSE\&G started notifying radio stations and news organizations about the rolling blackouts. However, the power shutdowns had already started at 7:02 a.m. and ended up affecting almost 400,000 customers (mainly homes and small businesses;. Many were upset about not being wamed. Complaints rolled in.

New Jersey Public interest Research Group, a public policy organization, denounced and questioned shutting off power to residences and small businesses. The Group conduded that this lack of communication was because "the public is not involved in the planning process regarding utility companies in New Jersey. Residential users are captive customers"

The acting director for rate counsel for the Public Advocate chimed in with, "We are trying to find out whether all the interruptable customers who pay a lower rate were off line prior to the rolling blackouts."

The PSE\& G spokesman responded that "The system is not designed so that only commercial customers would be shut off and not residential. It just depends what circuit a customer is on. We try not to shut off shopping centers and high-rise buildings because we would have to give them advance warning for safety reasons. "-MKC

prised of large motors which tend to draw more current as the voltage is decreased. If the current increases at a faster rate than the voltage decreases, this will result in a load power increase as the voltage drops.

On the other hand, residential loads, which tend to be heating related, are resistive in nature, and draw power which is proportional to $V^{2} / R$. In resistive loads, as the distribution voltage decreases, the real and reactive load demand will also decrease. This is due to the quadratic dependence of the load on the voltage. As the load drops, the power flow through the transmission lines will decrease, therefore the line loadings and reactive losses will also drop. Thus, residential low voltage problems tend to be "self-correcting." 


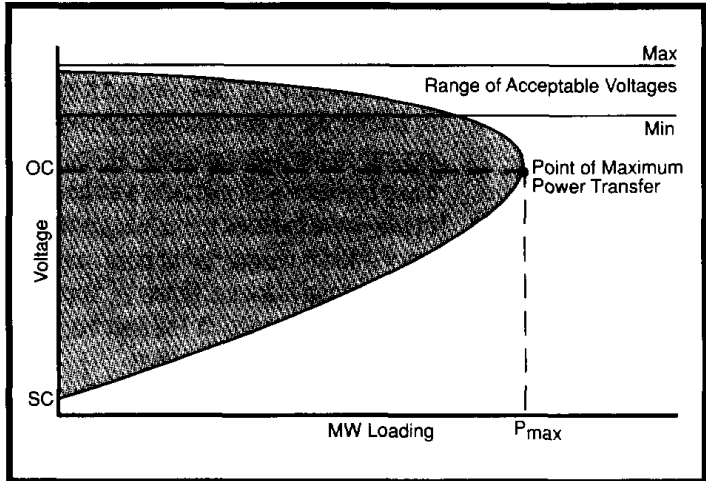

Fig. 1 Voltage as a symptom of heavy loading.

The high percentage of industrial loading as well as other conditions which may adversely affect voltage stability are listed for quick reference in Table 1.

An additional negative aspect of low voltages is the loss of reactive power

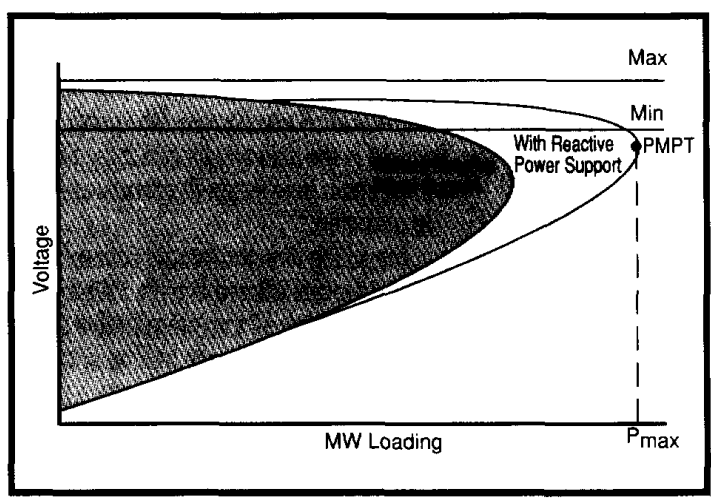

Fig. 2 Effect of installing controllable reactive support on system voltage.

support from shunt capacitor banks. Reactive power support helps maintain the voltage at a high level and increases the PMPT. However, as the voltage decreases, the amount of reactive power injected into the system by the shunt capacitors also decreases, further exacerbating the low voltage trend. This is because the reactive power is a function of $V^{2} / X_{c}$, where $X_{c}$ is the capacitive reactance of the capacitor bank.

Table 1

Conditions which may adversely affect voltage during a voltage collapse

- High Percentage of Industrial Loading

- Loss of Reactive Power Support

- Under-Load-Tap-Changing Transformers

- Generator Excitation Limits
Therefore, depending on the ratio of residential to industrial load in the system, and the amount of capacitive reactive support, the response of the combined loads may or may not balance. One direction tends to stabilize the system, the other direction leads towards voltage collapse.

Under-load-tapchanging (ULTC) transformers are used to maintain voltage levels by automatically adjusting their tap setting to raise or lower the distribution (or secondary) voltage on the demand side. Under most operating conditions, ULTCs are extremely useful control devices. However, during unusually low voltage conditions, the ULTCs may exacerbate the low voltage problem.

In this scenario, when the voltage on the transmission (or primary) side drops, the tap of the ULTC will adjust to keep the secondary voltage at the nominal operating level. This action will tend to keep the residential load at a high demand level, while affecting the industrial load only slightly. As the demand stays high, more current must be carried through the transmission to supply the load (due to the lower voltage), thus increasing the line losses. This causes a decrease in the transmission voltage, which will also decrease any shunt capacitor reactive support on the transmission side, causing a further decline in the transmission voltages.

If the taps of the transformers continue to adjust to keep the demand side voltage high, and subsequently the load, the transmission voltage will decrease continually until the taps reach their physical limits. At this point, the voltages on the demand side will start to decrease as well, possibly leading to a voltage collapse situation.

\section{Identification and correction}

In most cases, once the voltages have reached the point where they start to decrease rapidly, corrective actions are often too slow or are ineffective at averting the collapse. Therefore, most of the effort focused on voltage collapse has centered on predicting the occurrence of a collapse by finding an estimate, or index, of how close the system is to the critical point.

One of the most straightforward of these indices is the measure of the bus voltage sensitivity to changes in a load parameter. The motivation for this particular index comes from a study of the PV curve of Fig. 1. At normal operating voltages, there is very little change in the voltage level for an increase in load, thus the sensitivity of the voltage to changes in load is small. However, as the load increases and the voltages begin to decline, the voltage levels become increasingly sensitive to changes in load, until the PMPT when the voltage becomes infinitely sensitive to changes in the load.

This sensitivity concept may be extended to a large scale system. We can calculate a PV curve for each bus in the system, which will record that particular bus behavior as a function of system load increase. This methodology enables the utility system planner to isolate areas which exhibit large sensitivities. These areas are the regions in which voltage collapse is most likely to occur.

The PV curve of Fig. 1 is a twodimensional figure. It relates one bus voltage to the system real power load. Consider the possibility of expanding this curve to $n$-dimensions where all of the bus voltages are plotted against both real and reactive power. The critical PMPT now becomes a surface of maximum power transfer (MPT) in this ndimensional space. The point of system operation can now be located within this n-dimensional space. One serious disadvantage of the sensitivity index is that the value of the index does not change significantly until the system is already close to collapse.

A more accurate index of nearness to voltage collapse is a measure (in the $\mathrm{n}$-dimensional space) of the distance between the current system operating point and the surface of MPT. This ndimensional space is not actually physically represented, but may be represented mathematically. Because the index relates the entire system voltage levels to both real and reactive power; this index can provide useful information as to the amount and type of both real and reactive load which 


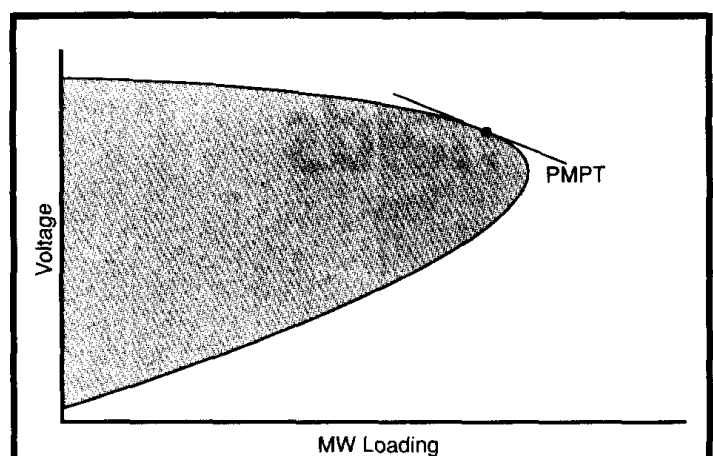

(a) Sensitivity related to slope

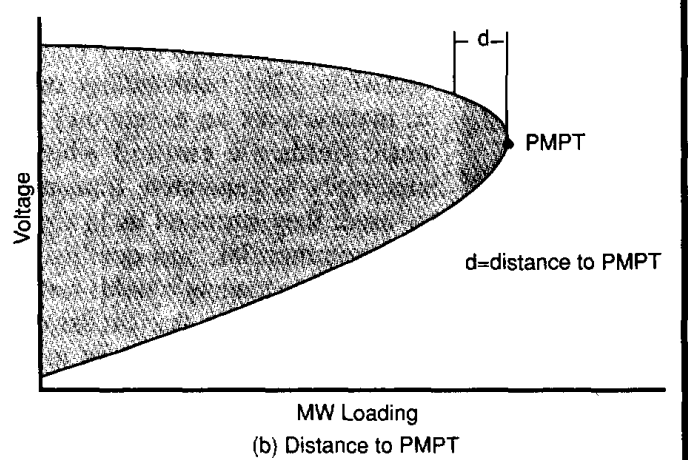

(b) Distance to PMPT

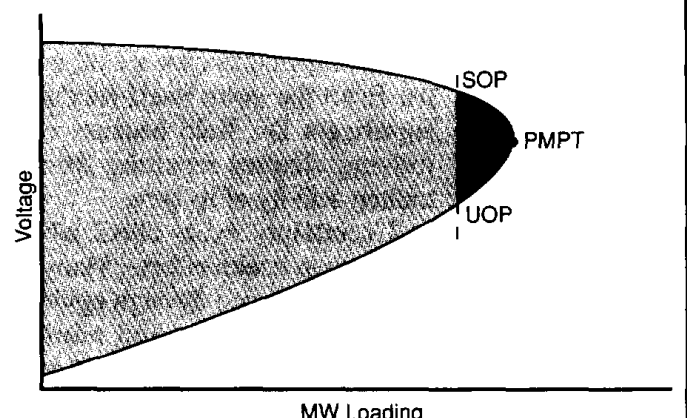

(c) Energy measure between SOP, UOP, and PMPT

Fig. 3 Comparison of voltage collapse indices.

could be shed if the system operating point moved too near the critical surface.

Another index which relies on the $\mathrm{n}$-dimensional representation is an energy index. Each operating point in the system is associated with a certain total energy (both potential and kinetic).

Referring once again to the PV curve of Fig. 1, note that each demand level is associated with two voltages. The upper voltage level is termed the stable operating point (SOP), the lower is called the unstable operating point (UOP). The critical PMPT occurs when these two operating points coalesce.
Another way of saying this, is that the energy difference between each of these two points becomes $\mathrm{O}$ when they coalesce.

This index, too, can be extended to the ndimensional space, where two energy measures are associated with each bus in the system, i.e. one for the stable condition, and a lesser energy for the unstable condition. This energy index tracks the entire system energy. A small or rapidly decreasing energy difference between the stable and unstable conditions indicates the potential for voltage collapse.

These three different indices are summarized in Fig. 3 for the simple, single line case. In Figure 3a, the sensitivity of voltage to changes in power is the slope of the PV curve at a given operating point. The distance to the PMPT is shown in Fig. 3b. The energy index is the difference between the energies at the SOP and UOP. This related to the shaded area shown in Fig. 3c.

\section{Conclusion}

The engineering challenge is to produce and provide electrical energy to consumers in a safe, economical and environmentally sound manner under increasingly stressed conditions. New, more accurate models are being developed to better predict how a realistic power system will react over a wide range of operating conditions. This work includes studies of how loads act dynamically.

New technologies are emerging to aid in the transmission of power. Among these, Flexible ac Transmission (FACTs) devices promise to be useful in the precise control of power flow through the network (see the article, "Flexible ac Transmission," in the April
1993 issue of the IEEE Spectrum). These have the potential of extending the transmission capabilities of the transmission network as well as aid in maintaining system stability. New control methods need to be developed and optimized to maintain accurate and stable control of the voltages and powers in power systems.

\section{Acknowledgments}

The authors would like to thank James L. Drewniak and E. Keith Stanek, University of Missouri-Rolla, and Peter W. Sauer, University of Illinois, for their useful comments and suggestions.

This work was supported in part by the National Science Foundation under grant ECS-9108914.

\section{Read more about it}

- Y. Mansour and P. Kundur, "Voltage Collapse: Industry Practices," C. T. Leondes (Ed.), Advances in Control \& Dynamic Systems, special volume on Analysis and Control System Techniques for Electric Power Systems, vol 42, part 2, pp. 111-162, 1991.

- System Dynamic Performance Subcommittee, "Voltage Stability of Power Systems: Concepts, Analytical Tools, and Industry Experience," IEEE Publication 90TH03582-PWR, IEEE, 1990.

- Energy Information Agency, Annual Energy Outlook 1993, Department of Energy publication DOE/EIA0383(93), January 1993.

- Edison Electric Institute, Electric and Magnetic Fields Task Force, Electric and Magnetic Fields: Summary of Developments, 1990.

\section{About the authors}

Mariesa L. Crow is an assistant professor in the electrical engineering department at the University of Missouri in Rolla. She was a recipient of the National Science Foundation Young Investigator award in 1992 and is a member of the IEEE Power Engineering Society System Dynamic Performance Subcommittee. Her primary area of research interest is computational algorithms, simulation and analysis of power system dynamics.

Bernard C. Lesieutre is an assistant professor in electrical engineering at the Massachusetts Institute of Technology. $\mathrm{He}$ is a member of Eta Kappa $\mathrm{Nu}$ and the IEEE. His research interests include machine modeling and power system dynamics, stability and control. 